livraisons

d'Histoire

de l'Architecture

\section{Livraisons de l'histoire de l'architecture}

$40 \mid 2020$

À propos des princes de Conti

\title{
Le concours pour la reconstruction du théâtre national de l'Opéra-Comique de 1893 : le concours public d'architecture, une solution à la crise architecturale de la fin du XIX ${ }^{\mathrm{e}}$ siècle?
}

Le concours pour la reconstruction du théâtre national de l'Opéra-Comique de 1893 : le concours public d'architecture, une solution à la crise architecturale de la fin du XIX siècle?

The competition for the reconstruction of the national theater of the OpéraComique in 1893: the public architectural competition, a solution to the architectural crisis of the end of the 19th century?

Der Wettbewerb zum Wiederaufbau des Théâtre national de l'Opéra-Comique 1893 - öffentlicher Architekturwettbewerb als Ausweg aus der Architekturkrise des ausgehenden 19. Jahrhunderts?

\section{Sven Peter}

\section{(2) OpenEdition}

Journals

\section{Édition électronique}

URL : http://journals.openedition.org//ha/1316

DOI : $10.4000 /$ /ha. 1316

ISSN : 1960-5994

Éditeur

Association Livraisons d'histoire de l'architecture - LHA

\section{Édition imprimée}

Date de publication : 3 décembre 2020

Pagination : 75-85

ISSN : $1627-4970$

\section{Référence électronique}

Sven Peter, «Le concours pour la reconstruction du théâtre national de l'Opéra-Comique de 1893 : le concours public d'architecture, une solution à la crise architecturale de la fin du XIXe siècle? ", Livraisons de l'histoire de l'architecture [En ligne], 40 | 2020, mis en ligne le 28 décembre 2020, consulté le 23 mars 2021. URL : http://journals.openedition.org//ha/1316 ; DOI : https://doi.org/10.4000//ha. 1316 
Par Sven PeTER

\section{LE CONCOURS POUR LA RECONSTRUCTION DU THÉÂTRE NATIONAL DE L'OPÉRA-COMIQUE DE 1893 : LE CONCOURS PUBLIC D'ARCHITECTURE, UNE SOLUTION À LA CRISE ARCHITECTURALE DE LA FIN DU XIX ${ }^{\mathrm{e}}$ SIËCLE ? ${ }^{1}$}

L'actuelle salle Favart, siège de l'Opéra-comique à Paris, est le résultat d'un concours de reconstruction qui fut lancé en 1893 après plusieurs années de tergiversations suite à l'incendie de la salle précédente en 1887. À l'issue du concours, le bâtiment que l'on connaît aujourd'hui fut finalement construit de 1893 à 1898 par l'architecte lauréat Louis Bernier (1855-1919).

L'histoire de la mise au concours et de la reconstruction proprement dite de cette salle ayant déjà été étudiée ${ }^{2}$, on s'attachera ici à une analyse du concours en lui-même : à la fois son organisation, son programme, les concurrents qui y participèrent, ainsi que des projets rendus, le jury et son jugement. En effet, de précieux documents restaient encore largement inexploités dans les collections publiques: en particulier des dessins des projets primés du concours au cabinet des estampes

1. Cet article est tiré de mon mémoire de master sous la direction de Basile Baudez à l'université Paris-Sorbonne: Sven Peter, "Le concours pour la reconstruction du théâtre national de l'Opéracomique de 1893 : le concours public d'architecture, une solution apportée à la crise architecturale de la fin du XIX ${ }^{\mathrm{e}}$ siècle ? ", mémoire de Master sous la direction de Basile Baudez, Paris, Université Paris-Sorbonne, 2017. Je remercie Pauline Chougnet et Basile Baudez de m'avoir proposé d'écrire cet article pour les Livraisons de l'histoire de l'architecture. Je remercie également Emmanuel Lamouche pour sa relecture attentive et ses précieux commentaires.

2. Denyse Rodriguez-Tomé, Un concours pour l'Opéra-comique? (1887-1893). Débats autour de la procédure de reconstruction d'un monument national. Le concours public en architecture, exercice de la démocratie et pratique professionnelle, mémoire [TPFE] sous la direction de Jean-Pierre Le Dantec, Paris, École d'architecture de Paris-la-Villette, 1997, traite de la genèse de la mise au concours ; Julie Charrier, La reconstruction du théatre national de l'Opéra-comique. Ou la genèse d'un projet éclectique (1893-1898), mémoire de $3^{\mathrm{e}}$ cycle [MES] sous la direction de Marc Deming, Paris, École nationale supérieure d'architecture de Paris-Belleville, 2006, s'attache à retracer la construction de la troisième salle Favart par Louis Bernier à partir des archives conservées de l'agence des travaux. Enfin, Marc Le Cœur, "L'Opéra-comique et sa reconstruction ", Béatrice de Andia, Géraldine Rideau (dir.), Paris et ses théâtres. Architecture et décor, Paris, Action artistique de la Ville de Paris, 1998, p. 103-110, s'est également penché sur la reconstruction de la troisième salle Favart, s'intéressant particulièrement aux débats de la mise au concours et à l'opposition entre des factions d'architectes. 
et de la photographie à la Bibliothèque nationale de France ${ }^{3}$, ainsi que des devis de projets, et les procès-verbaux de la commission et du jury du concours, conservés aux Archives nationales.

Dans l'historiographie, l'architecture de la fin du XIX ${ }^{\mathrm{e}}$ siècle est en général perçue comme une architecture en crise. Mais en quoi a pu consister cette crise ? Quel rôle y a pu jouer le concours public, réclamé par toute la profession des architectes, qui donna plus d'une fois des occasions ${ }^{4}$ de constater la crise d'une architecture désignée comme "académique " par ses détracteurs" 5 ? Plutôt qu'une solution à la crise architecturale, le concours public n'en serait-il pas une des sources?

\section{L'organisation du concours}

Au cours des six années qui ont suivi l'incendie de 1887, l'avenir d'une nouvelle salle pour le théâtre national de l'Opéra-comique demeura incertain ${ }^{6}$. Parallèlement à des plans de relogement de la troupe dans un autre théâtre, plusieurs projets de reconstruction furent proposés, mais aucun ne fut accepté définitivement. L'éventualité que le Conseil général des Bâtiments civils, administration en charge de la reconstruction, choisisse un projet proposé par des entrepreneurs, mobilisa et réunit les différentes associations d'architectes pour réclamer - avec succès - la mise au concours de la reconstruction du théâtre.

Une commission chargée d'arrêter le programme du concours fut instituée par arrêté ministériel du 21 mars $1893^{7}$. Les dix-sept membres proposés par le directeur des Bâtiments civils, Jules Comte (1846-1912), comprenaient des agents de l'État et des hommes politiques concernés par la reconstruction du théâtre, comme le directeur de l'Opéra-comique, Léon Carvalho (1825-1897), ainsi que cinq architectes du Conseil général, constitué par l'élite de la profession, dont Charles Garnier (1825-1898). L'importance de ce dernier dans l'organisation du concours de l'Opéracomique fut capitale : référence incontournable en matière d'architecture à l'époque,

3. Exception faite des dessins du projet de concours de Louis Bernier qui n'ont pas été transférés avec ceux des autres projets primés à la Bibliothèque nationale de France par le Conseil général des Bâtiments civils. Ils n'ont pu être retrouvés ni dans les fonds des Archives nationales qui conservent les versements des Bâtiments civils, ni dans les fonds de la Bibliothèque-musée de l'Opéra qui conserve les dessins de l'agence des travaux de Louis Bernier à l'Opéra-comique.

4. Parmi les plus fameux, on peut citer le concours pour la tour du Chicago Tribune de 1922, ou pour le siège de la Société des Nations à Genève de 1927. Voir par exemple Barry Bergdoll, "Competing in the Academy and the Marketplace ", Hélène Lipstadt (dir.), The Experimental Tradition. Essays on Competitions in Architecture, New York, Princeton Architectural Press, 1989, p. 23.

5. Voir par exemple Basile Baudez, Architecture \& tradition académique : au temps des Lumières, Rennes, Presses universitaires de Rennes, 2012, p. 310 sqq.

6. Voir Marc Le Cœur, "L’Opéra-comique et sa reconstruction ", op. cit.

7. République française, ministère des Travaux publics, Arrêté portant ouverture et réglant les conditions du concours pour la reconstruction du Théâtre national de l'Opéra-comique, Paris, Imprimerie nationale, 1893. 
et notamment d'architecture de théâtre ${ }^{8}$, Garnier était en outre directement en charge, en tant qu'inspecteur général du Conseil des Bâtiments civils, de la salle Favart ${ }^{9}$. Par décision unanime de la commission du concours, il fut chargé de l'élaboration du programme ${ }^{10}$.

Le concours à un seul tour, réservé aux architectes français, fut ouvert le 29 avril $1893^{11}$. Les concurrents avaient environ deux mois pour soumettre leurs projets, la clôture du concours ayant été fixée au 8 juillet. Bien que des études pour des projets de la reconstruction de l'Opéra-comique aient déjà été entamées depuis un moment, et que la commission en ait tenu compte en fixant une période relativement courte de deux mois, le programme n'exigea expressément que des avantprojets. Huit primes furent inscrites dans le programme. L'attribution de la commande au lauréat fut soumise à la condition que ce dernier élabore un projet définitif qui rencontre l'assentiment du Conseil général des bâtiments civils ${ }^{12}$.

Les contraintes du terrain étaient très importantes. Il se limitait à un rectangle de 52 mètres de long sur 30 mètres de large pour une salle de 1500 spectateurs, avec très peu de possibilité de recul sur la petite place Boieldieu, un immeuble mitoyen le séparant par ailleurs du boulevard des Italiens. En plus de plans, coupes et élévations, le programme exigeait également un devis descriptif ainsi qu'un devis sommaire devant évaluer le cube total de l'édifice et permettant ainsi de donner le prix de revient du mètre cube pour une dépense limitée à un maximum de 3500000 francs. Après le drame de l'incendie de l'ancienne salle, qui fit de nombreuses victimes, la sécurité en cas d'incendie fut par ailleurs un élément particulièrement important du programme.

\section{Les projets et leur jugement}

Quatre-vingts projets furent soumis au concours et exposés publiquement au Palais de l'Industrie, du 13 au 17 juillet 1893, avant leur examen par le jury ${ }^{13}$. Parmi les quatre-vingt-treize auteurs des projets connus ${ }^{14}$, un bon nombre étaient des architectes qui avaient de l'expérience dans la conception et la construction de

8. Ancien prix de Rome, son succès au concours pour la construction du Nouvel Opéra de Paris en 1861 lui avait assuré une carrière fulgurante. Voir par exemple Jean-Michel Leniaud, Charles Garnier, Paris, Monum-Éditions du Patrimoine, 2003, p. 49 sqq.

9. Charles Gourlier, Charles-Auguste Questel, Notice historique sur le service des travaux et sur le Conseil général des bâtiments civils, depuis la création de ces services en l'an IV (1795) jusqu'en 1895, Paris, Imprimerie nationale, 1895, p. 221.

10. Première séance, 25 mars 1893. Procès-verbaux des séances de la commission et du jury du concours pour la reconstruction du théâtre national de l'Opéra-comique, mars-juillet 1893: Paris, Arch. nat., $\mathrm{F}^{21} 6188$.

11. République française, ministère des Travaux publics, Arrêté portant ouverture..., voir n. 7.

12. Ibid.

13. République française, ministère des Travaux publics, ["Communication du ministère des Travaux publics concernant les conditions de la remise des projets ... »], Journal officiel de la République française, $\mathrm{n}^{\circ} 181,6$ juillet 1893, p. 3468.

14. Pour l'identification des concurrents, voir Sven Peter, Le concours ... de l'Opéra-comique de 1893, op. cit., t. I, p. 48-52 et t. II, p. 18-21. 
théâtres ; mais, plus remarquable encore est la participation très majoritaire d'architectes anciens élèves de l'École des Beaux-Arts. Une bonne partie d'entre eux constituait à cette époque l'élite des lauréats du Grand Prix de Rome, dont douze Premiers Grands Prix, quatre premiers Seconds Grands Prix et cinq deuxièmes Seconds Grands Prix. Bien que le concours ait été réclamé par toutes les associations professionnelles importantes de l'époque, la participation des architectes parisiens issus de l'École des Beaux-Arts et membres de la Société centrale, qui avait longtemps eu le monopole de la représentation de la profession, et surtout de la jeune Société des architectes diplômés par le gouvernement, prédominaient donc largement sur les architectes en dehors du système de l'architecture officielle ${ }^{15}$.

Ce caractère du concours de l'Opéra-comique se confirme également en ce qui concerne le jury. Aux membres de la commission, dont les cinq architectes membres du Conseil général Charles Garnier, Jean-Louis Pascal (1837-1920), Constant Moyaux (1835-1911), Honoré Daumet (1826-1911) et Émile Vaudremer (1829-1914), se joignirent cinq architectes élus par les concurrents conformément au programme : Ernest Coquart (1831-1902), Julien Guadet (1834-1908), Léon Ginain (1825-1898), Marcel Deslignières (1847-1914) et Paul Sédille (1836-1900) ${ }^{16}$. Pratiquement tous étaient ou avaient été professeurs chefs d'atelier à l'École des Beaux-Arts ${ }^{17}$. Il semble donc que la majorité des concurrents avait souhaité recréer une situation de jugement qu'ils avaient vécus comme étudiants. Bien que tous issus de l'École des Beaux-Arts de Paris, les architectes membres du jury étaient toutefois loin de représenter un courant uniforme d'architecture, mais reflétaient au contraire la diversité de l'architecture de l'époque ${ }^{18}$.

15. Ce système de l'architecture officielle en France au XIX ${ }^{\mathrm{e}}$ siècle est constitué d'institutions très intégrées : l'École et l'Académie des Beaux-Arts ainsi que les services d'architecture gouvernementaux, comme notamment les Bâtiments civils. Voir par exemple David van Zanten, "The Ecole, the Academy, and the French Government Architectural Services ", Jane E. Hargrove (dir.), The French Academy: classicism and its antagonists, Newark, University of Delaware Press, 1990, p. 196-205. Pour l'opposition professionnelle entre les associations d'architectes notamment " académiques » et " rationalistes " lors du concours, voir Marc Le Cour, "L’Opéra-comique et sa reconstruction ", op. cit., p. 107.

16. République française, ministère des Travaux publics, "Concours pour la reconstruction du théâtre national de l'Opéra-comique. Élection par les concurrents des cinq jurés complémentaires ", Journal officiel de la République française, $\mathrm{n}^{\circ} 183,6$ juillet 1893, p. 3602.

17. Charles Garnier n'eut jamais d'atelier d'élèves propre. Son agence des travaux à l'Opéra de Paris peut toutefois être comparée par son influence aux ateliers officiels. Voir Marie-Laure Crosnier Leconte, "L'agence de Charles Garnier à l'Opéra ", Charles Garnier : un architecte pour un empire, Bruno Girveau (dir.), cat. expo., École nationale supérieure des Beaux-Arts, Paris, 26 octobre 2010 9 janvier 2011, Paris, Beaux-Arts de Paris, 2010, p. 135 sq.

18. Ainsi, Émile Vaudremer fut proche des rationalistes et membre fondateur de l'U.S.A.F. en 1890. Il fut architecte diocésain mais également académicien. Constant Moyaux s'était également attaché à renouveler la tradition de l'architecture classique, en s'appropriant des idées rationalistes. Paul Sédille, bien que non officiellement associé aux rationalistes, se distingua quant à lui par ses recherches sur l'utilisation constructive et décorative du fer. Son ouvrage L'Architecture moderne en Angleterre contribua en outre à préparer le terrain à l'architecture moderne en France. Coquart, au contraire, était considéré comme un des chefs de l'école classique. Quant à Daumet, il était spécialiste d'une architecture inspirée de l'école française, notamment du XVI ${ }^{\mathrm{e}}$ siècle. Enfin, Pascal, tout comme Garnier, s'opposa à l'uniformisation internationale des styles en général, devenant l'un des 
Après la fin de la période d'exposition publique des projets du concours, le jury procéda au jugement. Les procès-verbaux des séances du jury révèlent le fait remarquable que les pièces annexes aux dessins des projets demandées par le programme, c'est-à-dire les devis descriptifs et estimatifs, furent rapidement écartées, le jury s'étant rendu compte qu'il était incapable de contrôler des données telles que le nombre de places que la salle pourrait contenir et le prix de revient du mètre cube $^{19}$. Le critère du budget en particulier, qui avait été tant discuté et dont le respect avait été mis en avant avec beaucoup d'insistance dans le programme, était ainsi omis du jugement, tout comme les aspects constructifs et techniques des projets. La sélection devait donc se faire exclusivement sur des critères relevant du dessin. Après plusieurs tours d'élimination et de sélection, le jury fit son choix : le projet de Louis Bernier reçut la première $\operatorname{prime}^{20}$. En plus des huit primes prévues par le programme, vingt-deux mentions furent distribuées à des projets qui n'avaient pas obtenu une prime, ceci afin d'exprimer l'importance du concours et la satisfaction générale du jury ${ }^{21}$.

\section{Résultats du concours et motifs du jury}

La commission s'était attachée particulièrement à rendre ses procédures plus transparentes, afin d'éviter les reproches régulièrement essuyés par le Conseil général des Bâtiments civils à l'époque. Outre l'élection de cinq jurés architectes supplémentaires par les concurrents eux-mêmes, et l'organisation d'une exposition publique, le programme stipulait la publication d'un rapport du jury "indiquant le motif de ses décisions » ${ }^{22}$. Ce rapport, rédigé par Julien Guadet, fut publié par le directeur des Bâtiments civils le 25 juillet dans le Journal officiel, et diffusé par la presse.

Les motifs que le rapport avança pour justifier le choix des projets primés révèlent les critères appliqués par le jury. Le premier critère pour justifier le choix du projet lauréat concernait la composition, qui peut être considérée comme l'essence de l'enseignement du projet d'architecture tel qu'il s'établit au cours du

précurseurs du régionalisme architectural en France. Voir Sven Peter, Le concours ... de l'Opéracomique de 1893, op. cit., t. I, p. 78-83. Ainsi, la diversité que représentaient les jurés architectes du concours illustre bien une caractéristique de l'École des Beaux-Arts, dont Julien Guadet définit l'originalité dans ses Éléments et théorie de l'architecture de 1901 comme "la plus libérale qu'il y ait au monde" (Julien Guadet, Éléments et théorie de l'architecture, Paris, Aulanier, 1901, t. I, p. 80).

19. Septième séance, 19 juillet 1893. Procès-verbaux des séances de la commission et du jury du concours pour la reconstruction du théâtre national de l'Opéra-comique, mars-juillet 1893 : Paris, Arch. nat., $\mathrm{F}^{21} 6188$.

20. Suivirent les projets d'Édouard Larche (1855-1939) et François Nachon (mort en 1909) (2e prime), de Paul Blondel (1847-1897) (3 ${ }^{\mathrm{e}}$ prime), de Gaspard André (1840-1896) (4e prime), d'Auguste Duvert (1828-1894) et Théodore Charpentier (1828-1902) ( $5^{\mathrm{e}}$ prime), de Pierre Esquié (18531933) (6 $6^{\mathrm{e}}$ prime), de Fernand Dupuis (1867-1930) ( $7^{\mathrm{e}}$ prime), finalement celui d'Adrien Chancel (1853-1901) ( $8^{\mathrm{e}}$ prime).

21. Jules Comte, "Rapport au ministre des Travaux publics ", Journal officiel de la République française, n 192,25 juillet 1893 , p. 3860-3861.

22. République française, ministère des Travaux publics, Arrêté portant ouverture..., voir n. 7. 


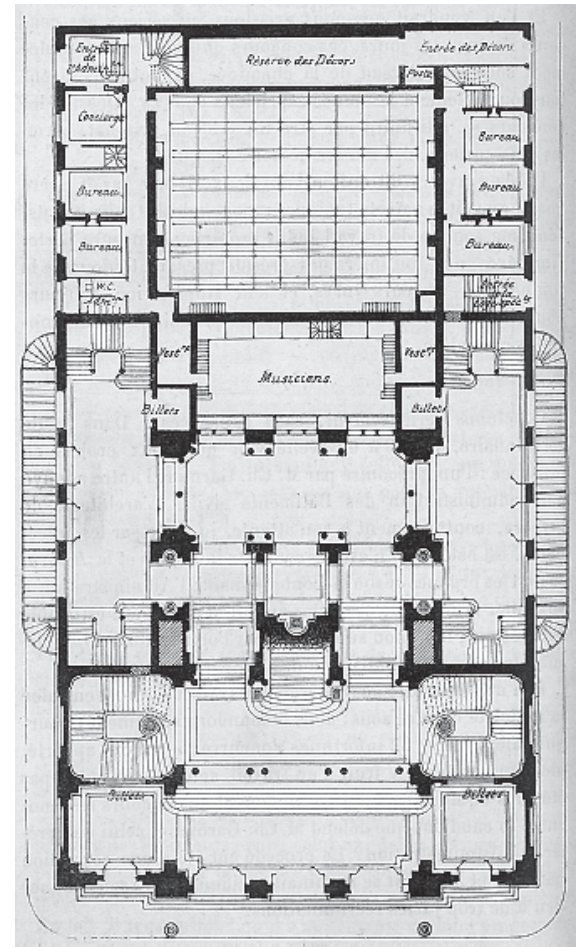

Ill. 1 : Louis Bernier (1845-1919), Projet pour la reconstruction du théâtre national de l'Opéra-comique, 1893, plan du rez-de-chaussée. (C) La Construction moderne, nº 30, 29 juillet 1893, p. 508.

$\mathrm{XIX}^{\mathrm{e}}$ siècle à l'École des Beaux-Arts ${ }^{23}$. Perçue comme la méthode incontestable de la conception et de la représentation du projet d'architecture à la fin du siècle ${ }^{24}$, la composition fut donc envisagée comme un critère particulièrement objectif. Dans le projet de Louis Bernier le jury reconnaissait ainsi « [...] avant tout une grande simplicité de disposition, une précieuse clarté des dégagements, une heureuse disposition des entrées et des sorties dans un plan de rez-de-chaussée ingénieux et habile ${ }^{25}$ (ill. 1). Le projet de Louis Bernier, également loué pour sa mesure et sa proportion, n'était pourtant pas prêt à être construit, un défaut ou une vertu dont le jury était bien conscient ${ }^{26}$ :

23. Jacques Lucan, Composition, non-composition. Architecture et théories, $X I X^{e}-X X^{e}$ siècles, Lausanne, Presses polytechniques et universitaires romandes, 2009, p. 101 sqq.

24. David van Zanten, "Architectural composition at the Ecole des Beaux-Arts from Charles Percier to Charles Garnier ", Arthur Drexler (dir.), The Architecture of the Ecole des Beaux-Arts, Londres, Secker \& Warburg, 1977, p. 112 sqq.

25. Jules Comte, "Rapport au ministre des Travaux publics ", op. cit., p. 3861.

26. Le Conseil général des Bâtiments civils, et en particulier Charles Garnier en tant qu’inspecteur général responsable de la salle Favart, intervinrent à plusieurs reprises avant qu'un projet définitif ne soit finalement accepté (Julie Charrier, La reconstruction du théatre national de l'Opéra-comique, op. cit., p. 188). 


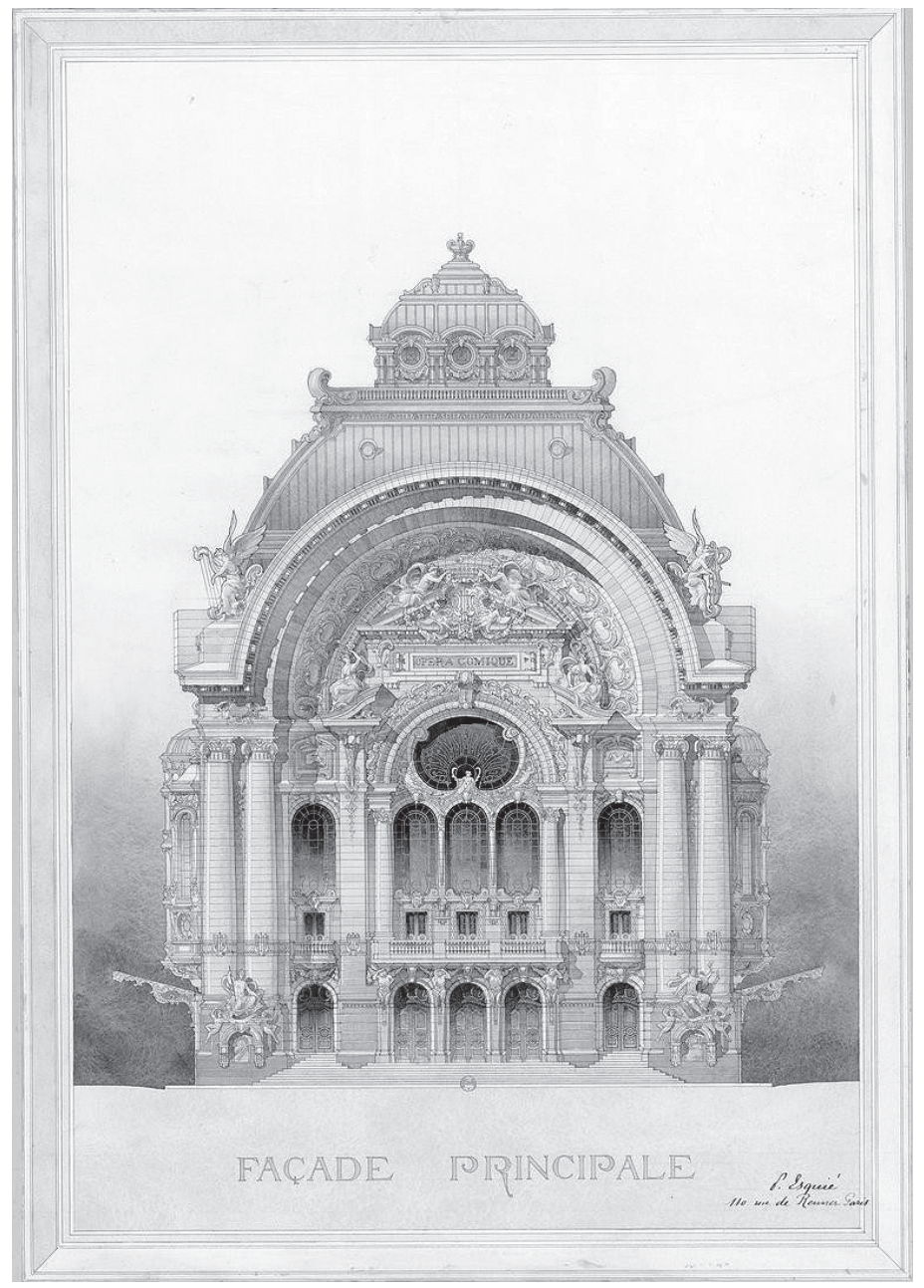

Ill. 2 : Pierre Esquié (1853-1933), Projet pour la reconstruction du théâtre national de l'Opéra-comique, 1893, élévation de la façade principale. (C) Bibliothèque nationale de France, Département des estampes et de la photographie, HA-86 (A)-FT 6.

«Mais le jury n'a garde d'oublier les dispositions fort sages de l'article 9 du programme du concours : il n'a pas entendu délivrer au projet de M. Bernier un certificat de parfaite solution, et il pense au contraire que dans la rédaction de son projet définitif cet artiste aura de nombreuses améliorations à apporter dans ses études [...]. En un mot, le concours n'avait à donner que des avant-projets, et c'est bien comme un avant-projet que le jury considère le projet de M. Bernier. ${ }^{27}$

27. Jules Comte, «Rapport au ministre des Travaux publics», op. cit., p. 3861. 


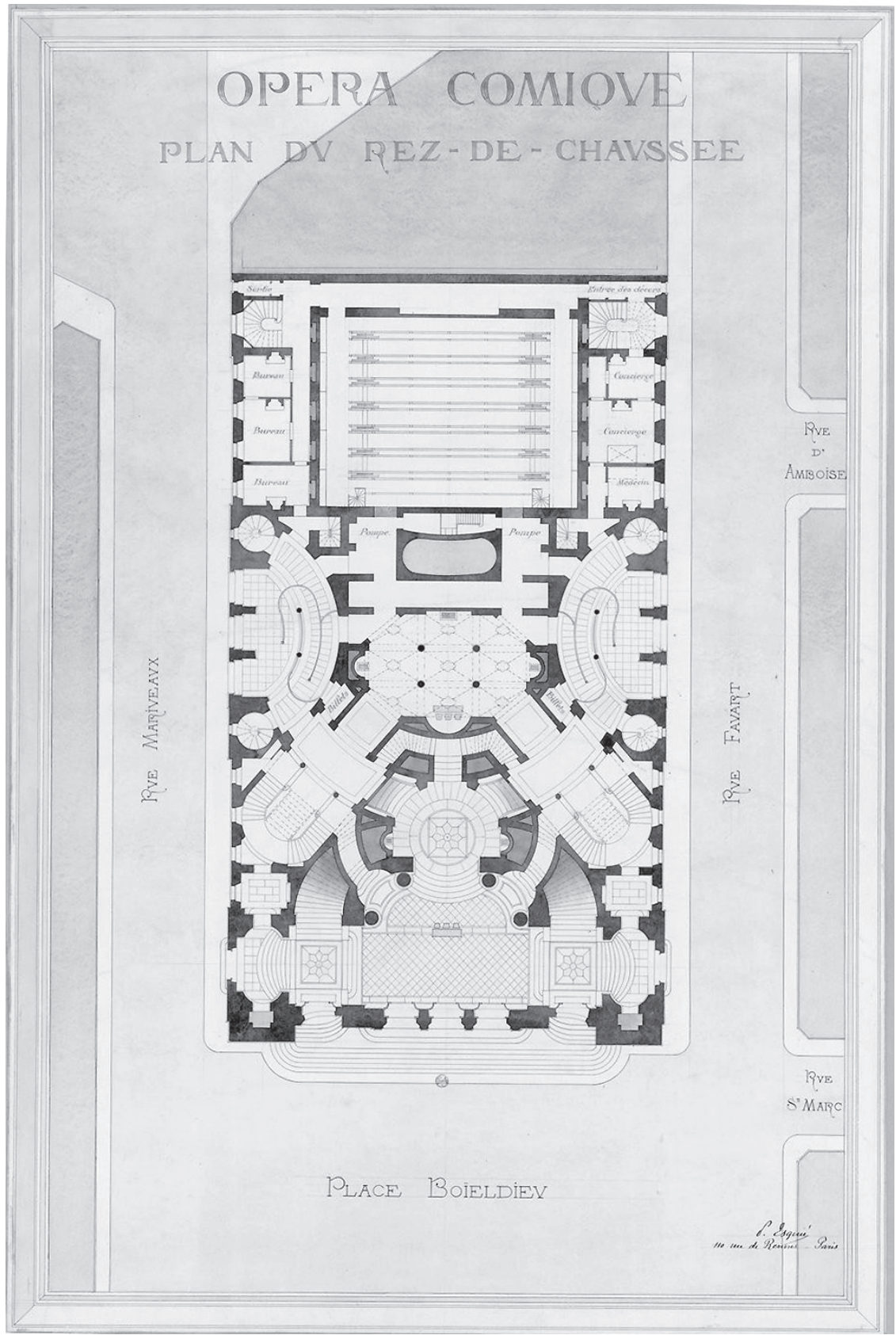

Ill. 3 : Pierre Esquié (1853-1933), Projet pour la reconstruction du théâtre national de l'Opéra-comique, 1893, plan du rez-de-chaussée. (C) Bibliothèque nationale de France, Département des estampes et de la photographie, HA-86 (A)-FT 6. 
La compétence artistique fut également un critère de première importance, qui pouvait se substituer à l'expérience pratique de l'architecte. Dans le cas de Louis Bernier, sa renommée artistique compensait ainsi son inexpérience pratique pour le jury qui affirmait : "[q]uant à l'étude artistique du monument, à l'intérieur et à l'extérieur, [...] on est en droit de compter sur la valeur de l'artiste, et qu'il est de ceux dont on espère avec confiance une œuvre d'art digne de l'État qui la lui confie et de Paris qui l'attend ${ }^{28}$. À mesure que le choix du jury s'éloignait de la sélection du projet lauréat, qui en tant qu' " esquisse " ${ }^{29}$ devait donc servir de schéma au projet définitif d'exécution du futur édifice, l'aspect artistique pouvait prendre le dessus sur le respect du programme. Le jury récompensa ainsi dans le projet de Pierre Esquié (ill. 2), un projet très remarqué dans la presse de l'époque et le «clou » du concours selon Émile Rivoalen, " une œuvre d'art d'une valeur telle qu'on oublie d'en relever les côtés critiquables au point de vue pratique " ${ }^{30}$. Mais ces "plans d'artiste ${ }^{31}$ ne pouvaient guère convenir à un programme de théâtre pour lequel la recherche de la sécurité était primordiale (ill. 3).

\section{Concours et crise}

Le jugement du concours se concentra ainsi sur les compétences liées au projet académique d'architecture, c'est-à-dire tel qu'il est enseigné et pratiqué à l'École des Beaux-Arts, et tel qu'il trouve son apogée dans les concours annuels du Grand Prix de Rome organisé par l'Académie des Beaux-Arts ${ }^{32}$, au détriment d'autres compétences du métier d'architecte découlant de la pratique professionnelle. Bien que le concours ait eu à l'origine comme objectif de choisir entre des architectes professionnels un candidat pour construire une nouvelle salle pour l'Opéra-comique, il semble donc que les questions pratiques, contenues avant tout dans les devis des projets, n'aient pas ou peu influé sur les décisions du jury. Certains projets étaient pourtant très élaborés et à la pointe du progrès technique et scientifique de l'époque ${ }^{33}$

28. Ibid. Grand Prix de Rome en 1872, Louis Bernier n'avait construit que l'hôtel particulier du peintre Léon Bonnat (1833-1922) à Paris avant d'obtenir la commande pour la reconstruction de l'Opéra-comique (Julie Charrier, Louis Bernier, architecte collectionneur bibliophile (1845-1919), mémoire [sans indication de diplôme ou de direction], Versailles, École d'architecture de Versailles, 2008).

29. C'est ainsi que furent désignés les avant-projets par Charles Garnier lors de la première séance du comité d'organisation du concours avant la rédaction du programme. Première séance, 25 mars 1893. Procès-verbaux des séances de la commission et du jury du concours pour la reconstruction du théâtre national de l'Opéra-comique, mars-juillet 1893, Paris, Arch. nat., F ${ }^{21} 6188$.

30. Émile Rivoalen, "Le Concours de l'Opéra-comique ", La Construction moderne, $8^{\mathrm{e}}$ volume, $\mathrm{n}^{\mathrm{o}} 29$, 22 juillet 1893, p. 494.

31. Ibid., p. 495.

32. Voir par exemple Richard Chafee, "The teaching of architecture at the Ecole des Beaux-Arts", The Architecture of the Ecole des Beaux-Arts, op. cit.., p. 61-109.

33. Le projet d'Édouard Bérard (1843-1912) prévoyait par exemple l'utilisation du ciment armé pour la construction de la nouvelle salle Favart, un procédé tout à fait nouveau à l'époque. Le devis, dactylographié et imprimé, qui est le seul à avoir été conservé par l'administration en dehors des devis des projets primés - ce qui montre l'intérêt que l'administration pouvait porter à ce nouveau 
ou révélaient une maîtrise de la conception et de la construction de théâtres ${ }^{34}$. Le jugement fut ainsi fortement critiqué dans la presse. Paul Planat (1839-1911), pourtant grand partisan du concours public ${ }^{35}$, exprima ainsi sa déception dans La Construction Moderne :

" Ainsi, on a convoqué, pour constituer un jury, les sommités de l'architecture, tous les membres de l'Institut, les hauts bonnets des Bâtiments civils, les représentants de la Chambre et du Sénat, des Beaux-Arts et des Travaux publics, la Préfecture de police et la Préfecture de la Seine; la presse a été mise en rumeur, le public est haletant; le concours est unanimement déclaré remarquable, il n'y a que l'embarras du choix entre plusieurs projets de premier ordre ; et cet Aréopage accouche d'un projet... inexécutable. La souris n'est même pas viable. » ${ }^{36}$

Le concours académique fournissait alors un modèle qui pouvait servir à réglementer la pratique du concours public ${ }^{37}$, mais dont la transposition pouvait également dénaturer le sens de ce dernier : au lieu de permettre une émulation entre des professionnels pour l'obtention d'une commande, l'adoption des procédures académiques transformait le concours public en une joute entre élèves. Alors que la pratique du concours académique était particulièrement élaborée en France ${ }^{38}$, la déception dans le jugement du concours de la reconstruction de l'Opéra-comique et les critiques qui visèrent ses procédures, montrent que le concours public à la fin du XIX ${ }^{\mathrm{e}}$ siècle n'était au contraire pas encore bien maîtrisé.

procédé de construction en général -, en témoigne. Édouard Bérard proposa «[un] mode de construction [...], nouveau en France, [...] employé à l'étranger où il a donné d'excellents résultats [...], l'on peut citer des exemples [...] notamment dans des établissements industriels » (Devis du projet du concours pour la reconstruction du théâtre national de l'Opéra-comique par Édouard Bérard, juillet 1893, Paris, Arch. nat., $\mathrm{F}^{21}$ 6187).

34. Gaspard André, par exemple, proposa dans son devis une adoption du "nouveau système allemand " à l'" ancien système français " de la salle du théâtre (Devis du projet du concours pour la reconstruction du théâtre national de l'Opéra-comique par Gaspard André, juillet 1893: Paris, BNF, Estampes, HZ-450 (1)-FT 7). La salle en amphithéâtre allemande se présentait comme une alternative à la salle à l'italienne - ou à la française - traditionnelle, et se référait au théâtre antique, avec sa salle semi-elliptique sans divisions, ce qui permettait un meilleur confort de vision et d'audition. Cette préoccupation s'exprimait en outre dans l'importance donnée à l'avant-scène et l'enfoncement de l'orchestre sous celle-ci. Voir par exemple Marc Bédarida, «L'amphithéâtre ou l'espace unifié : Wagner à Bayreuth et ses précurseurs ", Victor Louis et le théatre /: scénographie, mise en scène et architecture théatrale aux XVIII et XIX ${ }^{e}$ siècles, Paris, Éditions du Centre national de la recherche scientifique, 1982, p. 177-186.

35. Denyse Tomé-Rodriguez, Un concours pour l'Opéra-comique?, op. cit., p. 7.

36. Paul Planat, "Moralité du concours de l'Opéra-comique ", La Construction moderne, $8^{\mathrm{e}}$ volume, $\mathrm{n}^{\circ} 30,29$ juillet 1893 , p. 505-507. Planat était d'ailleurs loin d'être le seul à critiquer le résultat du concours. Même le supposé maitre du concours pour la reconstruction de l'Opéra-comique, Charles Garnier, exprima dans un entretien accordé à Le Figaro son mécontentement dans le résultat du jugement, dont l'annonce avait pourtant semblé témoigner l'unanimité du jury. Ibid., p. 506.

37. Barry Bergdoll, "Competing in the Academy and the Marketplace", The Experimental Tradition, op. cit., p. 43.

38. Voir ibid., p. 21-51. 
Entre l'annonce de la mise au concours de la reconstruction de l'Opéra-comique et son ouverture officielle, Julien Guadet avait d'ailleurs publié une suite d'articles sur la procédure du concours public ${ }^{39}$, qu'il terminait sur ces mots :

«Tel est l'exposé général des idées théoriques que fait naître la question des concours publics. On voit par là combien cette question a été, en réalité, peu étudiée ; on se guide par le hasard des bonnes intentions, on tâtonne, et il n'y a guère, dans la pratique des concours, que confusion et contradictions. Et cependant les concours publics répondent à une même pensée, découlent d'un principe constant; il y a évidemment une méthode qui est la meilleure et qui devrait être appliquée partout, par toutes les administrations; il ne leur manque, pour cela, que de la connaître. ${ }^{40}$.

Comme le démontre l'étude du concours pour la reconstruction de l'Opéracomique, le concours public, bien que souhaité par toute la profession, ne permit pas un accès égalitaire à la commande publique à tous les architectes. Toutefois, les procédures de l'État concernant l'attribution de la commande en furent rendues plus transparentes : l'État s'engageait directement envers l'opinion publique par ses publications officielles dans la presse et l'exposition publique des projets.

Partant d'une crise professionnelle, c'est-à-dire l'indécision sur le choix d'un architecte à reconstruire un théâtre, l'adoption du concours public révéla donc à toute la profession et au public la difficulté de trouver des critères adaptés à l'évaluation et à la sélection du projet d'architecture. La réduction de celui-ci, induite par l'adoption du concours académique comme modèle, masque ainsi l'innovation et la diversité qui caractérisent les projets d'architecture de la fin du XIX ${ }^{\mathrm{e}}$ siècle. Ainsi la transposition des critères du concours académique au concours public ne résolut pas la crise professionnelle, mais renforça au contraire le sentiment d'une crise de l'architecture.

Sven PETER

Master of sciences, université de Bamberg (Allemagne) Master of Arts, Université de Paris Sorbonne

39. Julien Guadet, "Les concours publics d'architecture. Étude ", L'Architecture, nº 11, 18 mars 1893 , p. 111-114 et du même, "Les concours publics d'architecture. Étude ", L'Architecture, no 12, 25 mars 1893, p. $122-124$.

40. Ibid., p. 124. 
